Relations industrielles

Industrial Relations

\title{
Management in the Industrial World: An International Analysis. By Frederick Harbison and Charles A. Myers. New York, McGraw-Hill, 1959, 413 pp., \$7.00.
}

\section{Roger Chartier}

Volume 15, numéro 1, janvier 1960

URI : https://id.erudit.org/iderudit/1022083ar

DOI : https://doi.org/10.7202/1022083ar

Aller au sommaire du numéro

Éditeur(s)

Département des relations industrielles de l’Université Laval

ISSN

0034-379X (imprimé)

1703-8138 (numérique)

Découvrir la revue

Citer ce compte rendu

Chartier, R. (1960). Compte rendu de [Management in the Industrial World: An International Analysis. By Frederick Harbison and Charles A. Myers. New York, McGraw-Hill, 1959, 413 pp., \$7.00.] Relations industrielles / Industrial Relations, 15(1), 146-147. https://doi.org/10.7202/1022083ar

Tous droits réservés @ C Département des relations industrielles de l’Université Laval, 1960
Ce document est protégé par la loi sur le droit d'auteur. L'utilisation des services d'Érudit (y compris la reproduction) est assujettie à sa politique d'utilisation que vous pouvez consulter en ligne.

https://apropos.erudit.org/fr/usagers/politique-dutilisation/ 
technique. La seconde, « Problèmes du développement », envisage: de l'économie de subsistance à l'économie de marché; économie harmonisée et planification; politique des investissements; enseignement technique et recherche scientifique. L'auteur donne des exemples concrets, de façon à permettre de comprendre mieux les problèmes à résoudre. Les annexes sont consacrées à fournir les renseignements utiles sur les divers organismes qui s'occupent de cette question. Enfin, l'ouvrage est agrémenté de plusieurs photographies.

\section{GÉrard Dion}

Management in the Industrial World: An International Analysis. By Frederick Harbison and Charles A. Myers. New York, McGraw-Hill, 1959, 413 pp., \$7.00.

Cette analyse comparative de la nature de la direction des entreprises industrielles est le fruit de l'effort combiné des Sections de relations industrielles de Princeton et du M.I.T. Elle s'appuie sur plusieurs études monographiques, préparées par les auteurs et par six autres collaborateurs, de la direction industrielle en Inde, en Egypte, au Chili, en Israël, en France, en Italie, au Japon, en Allemagne, en Suède, en Grande-Bretagne et en Union soviétique. Cette série de monographies constitue la seconde partie du volume.

Chaque auteur, en son lieu, décrit le contexte et le processus de l'industrialisation du pays en cause, brosse un tableau réaliste de l'«élite» directoriale, examine la direction des entreprises comme système d'autorité, comme ressource et comme classe, en évalue la qualité et la puissance et indique les moyens de sa perpétuation.

Sur cette base solide, les deux auteurs principaux ont beau jeu, en première partie, pour fixer les contours d'un concept international de la direction 》 qui se réfère aux catégories précitées: la direction perçue comme ressource économique, comme système autoritaire et comme classe sociale.

En dernier lieu, les auteurs précisent la dynamique de la direction ainsi que la logique de ce développement. Ils confrontent les données internatio- nales ainsi acquises avec la réalité de la direction des entreprises aux EtatsUnis, terminant par une étude des problèmes de la direction des entreprises étrangères dans les pays économiquement sous-développés.

Cette oeuvre lucide est originale par plus d'un point. C'est une des premières fois, croyons-nous, qu'on s'est attaché aussi systématiquement à une comparaison internationale de la réalité directoriale dans l'optique du développement économique. Les auteurs ont le mérite de distinguer la direction-ressource du capital-ressource et d'indiquer clairement l'importance de cette distinction pour toutes les économies industrielles qui sont en train de former leurs élites directoriales.

La dynamique de l'industrialisation apparaît avec beaucoup de clarté dans la description d'économies qui se situent à des stades différents d'industrialisation. De même apparaissent clairement les diverses philosophies, l'éventail des structures et l'évolution variable de la direction dans divers pays.

Mais au delà des divergences, il semble exister comme une \&logique ? de l'évolution de la direction des entreprises centrée sur l'industrialisation; c'est ce modèle «universel 》 que les auteurs se sont efforcés d'abstraire de la réalité multiforme et de faire ressortir dans leur ouvrage. Ainsi, trois propositions générales semblent s'appliquer à toutes les sociétés industrielles: 1) \& The general direction of management development in all advancing industrial societies is the same $\gg ; 2$ ) \& The pace of the march toward industrialism may be accelerated or retarded by certain factors affecting management development 》; et 3) 《The$r e$ is little reason to fear that the working masses in modern industrial states will be exploited by the emerging professional managerial class $\gg$.

Voici donc un volume lumineux, neuf à plus d'un égard, qui marque un pas en avant dans l'analyse des \& élites $\gg$ directoriales des entreprises industrielles. Il devrait susciter une vive et utile discussion chez tous ceux (membres de la direction, chefs syndicaux, administrateurs gouvernementaux et professeurs d'université) qu'in- 
téresse le phénomène global de l'industrialisation et la définition du rôle de la direction dans le développement at le déroulement de ce phénomène.

\section{Roger Chartier}

\section{La liberté syndicale, cours d'éducation} ouvriere, Genève, Bureau international du Travail, 1959, 166 pp. (202, rue Queen, Ottawa 4, Ont.) prix: 75 cts.

Le Bureau international du Travail a entrepris la publication d'une série de cours qui s'inscrivent dans le cadre du programme d'éducation ouvrière de l'Organisation internationale du Travail.

Cet ouvrage est consacré à l'importante question de la liberté syndicale. Il est composé de dix-huit leçons groupées en quatre parties. $\mathrm{La}$ première partie retrace à grands traits les étapes principales de l'évolution du droit syndical. La deuxième décrit le rôle joué par l'Organisation internationale du Travail dans le domaine de la liberté syndicale et de la protection du droit syndical. Dans les troisième et quatrième parties, on a examiné un peu plus longuement l'état présent de la liberté syndicale dans les diverses parties du monde. Enfin, dans la dernière leçon, qui constitue la conclusion du cours, les auteurs ont essayé de dégager quelques enseignements de lexamen du problème.

Cet ouvrage se contente de présenter un historique et d'exprimer la situation actuelle en ce qui concerne les libertés syndicales et la protection du droit syndical. Il n'est pas une étude complète de ces questions, mais, en plus d'être une excellente initiation, le plan qu'il utilise peut être très précieux pour ceux qui ont des cours à donner sur le sujet.

Nous recommandons fortement cet ouvrage à tous ceux qui ont à s'occuper d'éducation syndicale.

\section{G. D.}

Job Attitudes: Review of Research and Opinion by $\mathrm{F}$. Herzberg \& al, Pittsburgh, Psychological Service of Pittsburgh, 1957.
Ce volume a pour fin d'étudier, d'organiser et de résumer les travaux publiés depuis 1954 sur le moral et les attitudes des employés envers leur travail. Chaque chapitre se termine par un résumé suivi de questions très pertinentes et de suggestions pour fins de recherche.

Tout d'abord, les auteurs font une revision des analyses sur les caractéristiques des employés mécontents de leur travail: âge, années de service, sexe, éducation, intelligence, personnalité, etc.

Le moral des employés est relié à plusieurs facteurs ou aspects du travail. Pendant les enquêtes, ses divers facteurs sont évalués sous forme de questions différentes quant à la forme, mais semblables quant au contenu. Ici les auteurs ont groupé tous les aspects du travail reliés au moral en dix facteurs élémentaires. Ces facteurs ont une importance relative qui varie selon les circonstances et les conditions de travail. Les auteurs concluent leur étude sur les facteurs en exposant de quelle façon ils sont reliés aux caractéristiques des travailleurs. Leurs suggestions poir fins de recherche sont très originales. Selon eux, il serait bon à ce stage d'utiliser les résultats obtenus directement dans l'industrie, afin d'en vérifier les effets sur la production, le roulement de la main-d'oeuvre, et le moral en général.

Les auteurs abordent également un sujet des plus contreversés: les conséquences directes du moral sur la production. l'absentéisme, le roulement de la main-d'oeuvre, les accidents industriels, la maladie, etc. Les auteurs citent au moins quatorze recherches qui semblent indiquer que le moral des employés est relié positivement à la production. Toutefois, si l'on examine bien les indices de corrélation rapportés dans ces recherches, il est évident que ces corrélations, quoique statistiquement significatives, sont très faibles. La plupart des chercheurs ne rapportent en général aucun indice de validité et de fiabilité sur l'instrument qui a servi à mesurer le moral des employés. Il est bien difficile d'estimer jusqu'à quel point les corrélations sont contaminées, car dans les circonstances il se peut fort bien qu'elles soient dues au hasard. Plus les corrélations sont fai- 